\title{
Monitoring the fatigue crack on the test specimen during the cyclic loading
}

\author{
Milan Sapieta ${ }^{1, *}$, Peter $\breve{S} \operatorname{lka}^{1}$, Martin Svoboda $^{2}$ \\ ${ }^{1}$ Faculty of Mechanical Engineering, Univerzity of Žilina. Unierzitná 1, 01026 Žilina. Slovak \\ Republic \\ ${ }^{2}$ Faculty of production technology and management of Jan Evangelista Purkyně University in Ústí \\ nad Labem, Na Okraji 1001, Czech Republic
}

\begin{abstract}
The main purpose of this paper is monitoring propagation of plastic area of fatigue crack. There were made notch on specimens and they were cyclically loaded with three-point bending. The record was evaluated after a certain number of loading cycles. The loading force is still constant throughout the measurement time for all specimens. The whole process was recorded using an infrared camera.
\end{abstract}

Keywords: crack propagation, cyclic load, infrared, radiation.

\section{Introduction}

Nowadays, a combination of numerous infrared detectors or thermal sensors with advanced signal processing generates a practical tool for investigating thermal-stress characteristics of materials and structures from the respective surface or part of the space.

Thermoelastic stress analysis has been used by engineers and scientists for more than 50 years to address practical problems. It utilises the principle of sensing the energy released during loading in the elastic domain. Once loading is removed, the body returns to its original position (elasticity) and its original temperature (thermoelasticity) [1].

\section{Thermoelasticity and temperature variation}

Thermoelastic stress analysis (TSA) is an experimental contactless method based on measuring the infrared radiation emitted from the component surface exposed to dynamically linear elastic strain (deformation). Kelvin was the first scientist to study the thermoelastic effect, and the basic equations to describe the thermoelastic [2] effect were formulated by Darken and Curry.

The general form of the heat conduction equation for elastic body is derived from the energy conservation equation, and it can be written as follows:

$$
\rho c_{\varepsilon} \frac{d T}{d t}-\frac{\partial}{\partial x_{j}}\left(k \frac{\partial T}{\partial x_{j}}\right)=\rho r+\sigma_{i j} \frac{\partial \varepsilon_{i j}}{\partial t}-\rho \frac{\partial \Psi}{\partial V_{k}} \frac{d V_{k}}{d t}+\rho T \frac{\partial^{2} \Psi}{\partial T \partial V_{k}} \frac{d V_{k}}{d t} .
$$

\footnotetext{
*Corresponding author: milan.sapieta@,fstroj.uniza.sk

Reviewers: Juraj Gerlici, Milan Nad'
} 
Equation (1) uses the Einstein summation convention, $\rho$ is the density, $\boldsymbol{c}_{\boldsymbol{\varepsilon}}$ is the specific heat capacity at constant deformation, $\boldsymbol{T}$ is the absolute temperature, $\boldsymbol{k}$ is the thermal conductivity tensor, $\boldsymbol{\sigma}_{\boldsymbol{i} \boldsymbol{j}}$ and $\boldsymbol{\varepsilon}_{\boldsymbol{i} \boldsymbol{j}}$ are the tensors of stress and strain, $\boldsymbol{\varepsilon}_{\boldsymbol{i j}}$ is the inner heat source per unit of volume and $\boldsymbol{\Psi}$ is Helmholtz free energy that is dependent on $\boldsymbol{k}$ and independent of the internal state variables $\boldsymbol{V}_{\boldsymbol{k}}$.

Provided that the material elastic properties and constant material coefficients are temperature independent, then it can be derived a 3-dimensional heat conduction equation written as follows:

$$
\rho \mathrm{C}_{\varepsilon} \dot{\mathrm{T}}-\mathrm{k} \Delta^{2} \mathrm{~T}=\mathrm{T}_{0}\left(-\frac{\mathrm{E} \alpha}{1-2 \mathrm{v}}\right) \dot{\varepsilon}_{\mathrm{I}}^{\mathrm{e}}+\alpha_{\mathrm{p}} \sigma_{\mathrm{ij}} \dot{\varepsilon}_{\mathrm{ij}}^{\mathrm{p}}
$$

The equation for heat conduction will include the creation of the thermoelastic and the thermoplastic heat. Parameter $\varepsilon_{\mathbf{I}}^{\mathbf{e}}$ denotes the first invariant of elastic deformation tensor $\boldsymbol{\varepsilon}_{\mathbf{i j}}^{\mathbf{p}}$ is the plastic part of the deformation tensor, $\alpha$ is the coefficient of thermal expansion, $\mathrm{E}$ Young's modulus, $v$ Poisson's ratio and $\mathbf{T}_{\mathbf{0}}$ is the initial temperature [3]. Non-dimensional coefficient $\alpha_{\boldsymbol{P}}$ is the ratio of the total plastic work to plastic work, which is converted to heat. The value $\boldsymbol{\alpha}_{p} \cong \mathbf{1}$ because of only a small part of the plastic work (obtained energy at cold forming) is used to change the inner properties of the material [4]. Therefor the thermoplastic area will be neglected and relationship will be formulated only for thermoelastic area, which has the following form:

$$
\Delta \mathrm{T}=\frac{\alpha \mathrm{T}_{0}}{\rho c_{\mathrm{p}}} \Delta \sigma_{\mathrm{ii}}
$$

where $\mathbf{c}_{\mathbf{p}}$ is the specific heat capacity under constant pressure and its relationship with $\mathbf{c}_{\boldsymbol{\varepsilon}}$ is as follows:

$$
c_{\varepsilon}=c_{p}-\frac{2 E \alpha^{2} T}{\rho(1-v)}
$$

\section{Measurement setup}

Within this work it was carried out measurement of three-point bending of stainless steel beams. The specimen was $50 \mathrm{~mm}$ long and had a 10x10 mm square cross section. For this measurement it was chosen three types of material, namely: AISI 304, AISI 316L, and AISI 316TI.

The specimen was put on two supports and the loading forces acted from above onto the specimen centre between the supports Fig. 1. The supports were placed apart at a distance of $29 \mathrm{~mm}$. 


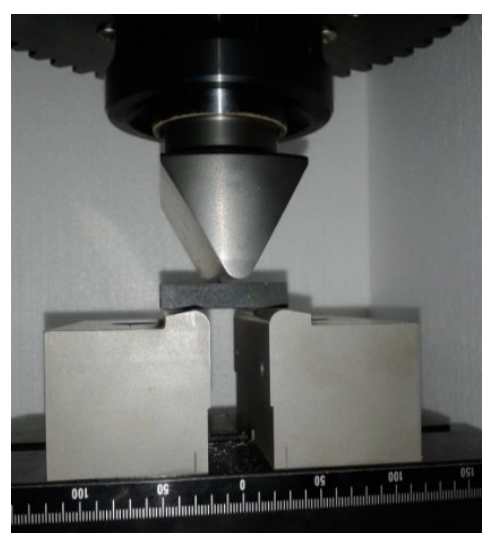

Fig. 1. Specimen position during the three-point bending loading

The notch width of $0.8 \mathrm{~mm}$ and a depth of $0.5 \mathrm{~mm}$ were created on the specimen. This notch was placed at the center of the bottom side of the specimen.

Again, it was chosen the infra-red camera maximum resolution 320x256 pixels, and corresponding frame rate $383 \mathrm{~Hz}$. Loading frequencies differed for each material and each loading state - they fluctuated in the range of 100-105 Hz. Once again there was a slight subsampling. Since it was found from the previous measurements that this type of subsampling does not affect the quality of results, it chose that as the most suitable one in view of the resolution - sampling frequency ratio [5-7].

The intermediate ring was inserted between the camera and its lens. The intermediate ring extends the focal length of the lens to increase the view of the subject. Basically, the resolution of the camera remains $320 \times 256$ pixels, it only changes the overall frame size (decrease), the FOV and IFOV parameters. Taking advantage of the intermediate ring also brings its hardship and is not always possible to use it. As a result of its use, the depth of field also changes, making it necessary to place the camera closer to the subject, which is not always possible in terms of safety. It is very easy to damage the lens, it is not economically advantageous due to lens cost.

The load was chosen constant for all 3 types of materials, the static load component was $6.5 \mathrm{kN}$ and the dynamic $6 \mathrm{kN}$. Specimens were cyclically loaded with frequency $105 \mathrm{~Hz}$ and were attempting to record after a certain number of cycles. Recording was done after 5000 cycles.

\section{Evaluation of measured results}

As a result of the AISI 304 and AISI 316L specimens not having suffered enough cycles (AISI 30413780 cycles and AISI 316L 6923 cycles), it was not possible to carry out a sufficient number of records to track fatigue crack propagation. It will further be evaluated formation and propagation of fatigue cracks only sample material AISI 316Ti. With this specimen, the fatigue fracture was up to 24,149 cycles, so up to 6 records were made.

The records were evaluated in unmodified DL (digital level) units. For maximum sensitivity, the lock-in method and the thermoelastic analysis were used. In this case, it is possible to determine the stress after calculating the temperature data, but only at the present time relationships are formulated for the elastic area (near the crack root is the expected plastic area). Therefore, the following observations were evaluated only quantitatively. 
The first record was performed at the beginning of the load after a certain number of cycles, essentially as soon as the machine was able to stabilize the load frequency. The first record shows the distribution of radiated radiation in DL units Fig. 2.

Figure 2 shows the distribution of radiation energy where negative values can be observed at the top of the sample, which is the stress of the longitudinal fibers to the pressure and the positive values at the lower part representing the tensile of the longitudinal threads.

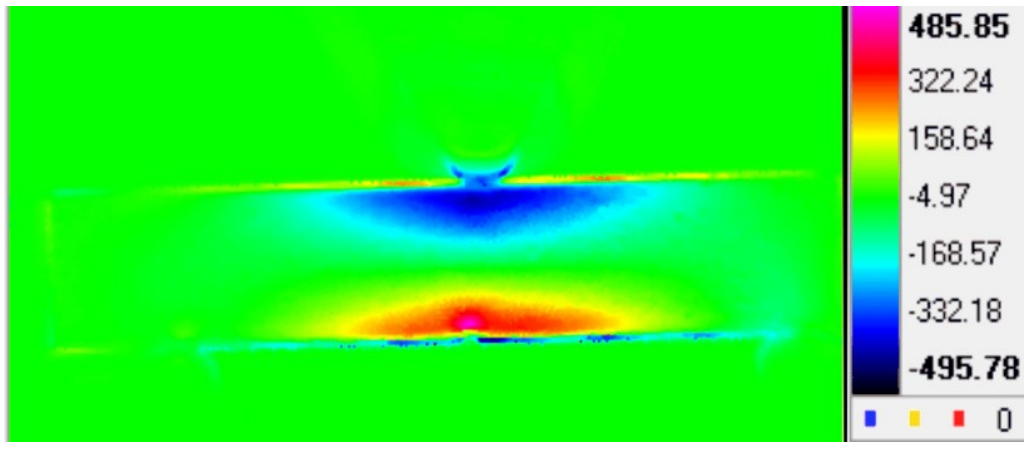

Fig. 2. Distribution of radiation energy recorded by the IR camera at the beginning of the fatigue test

The second record was performed approximately after 5000 cycles. As a result of the increase of the maximum value of the radiation energy, there was a scaling (this explains the background colour change, where the value should be zero). There is an increase in the upper and lower areas, but what is more important in the lower part above the notch are visible, the first signs of crack formation (purple) Fig. 3.

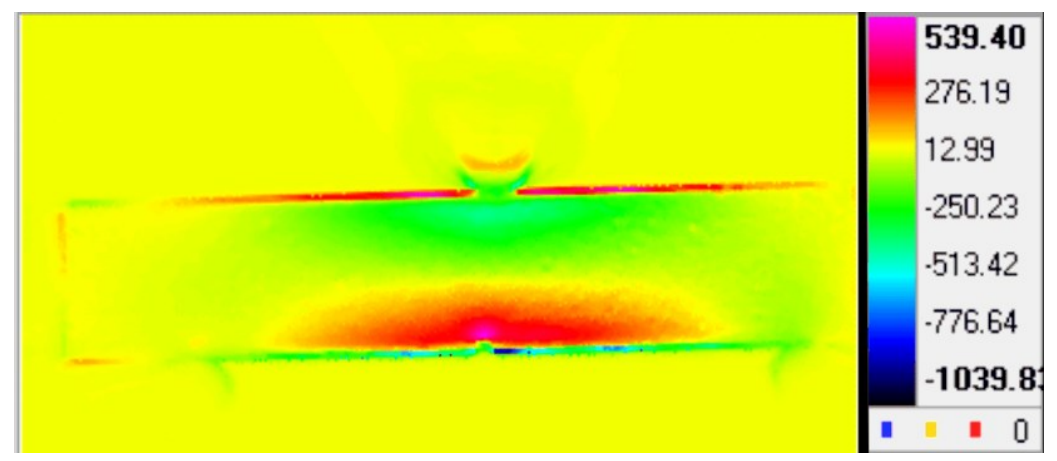

Fig. 3. Distribution of radiation energy recorded by the IR camera after 5000 cycles, the first signs of fatigue cracking

After 10,000 cycles, a third record was taken. There is a clearly visible crack in the image just above the notch Fig. 4. The crack is purple and this colour will be characteristic of this crack throughout the measurement time. Again, assuming an increase in the radiation values, depending on the number of cycles. 


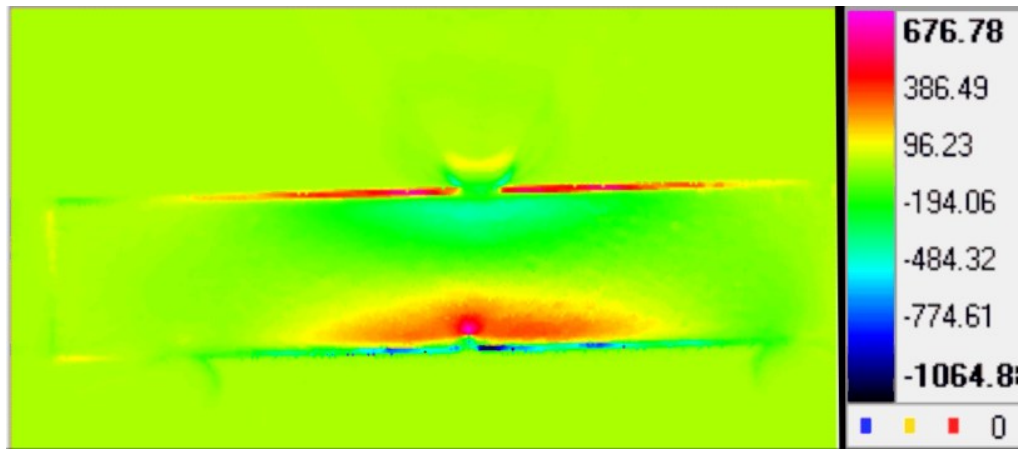

Fig. 4. Distribution of radiation energy recorded by the IR camera after 10000 cycles

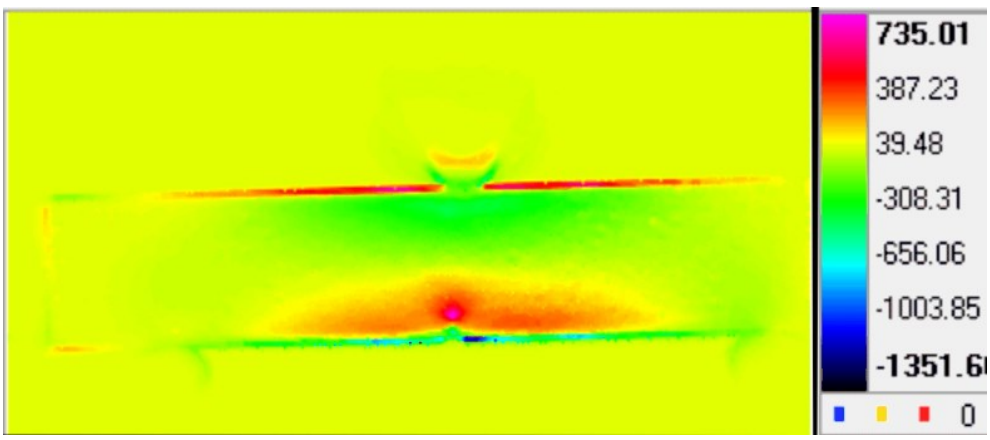

Fig. 5. Distribution of radiation energy recorded by the IR camera after 15000 cycles

The crack retains its size, it can be observed its gradual shift towards the centre of the specimen Fig. 5, 6.

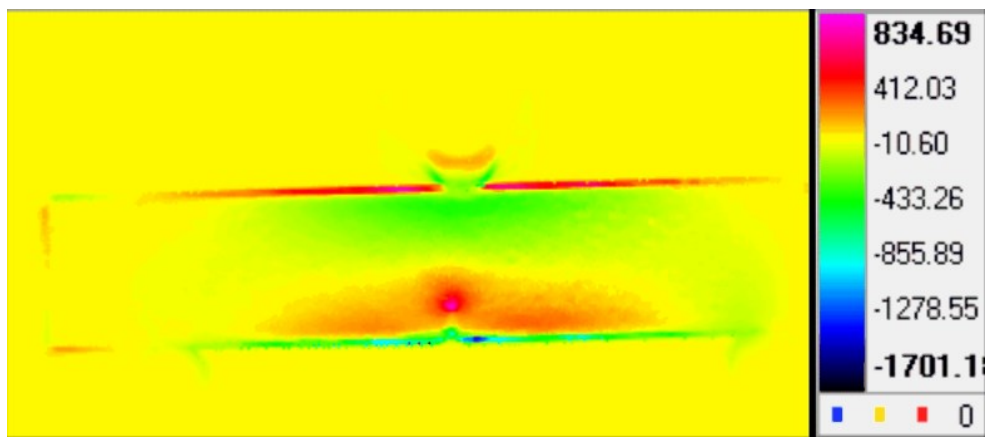

Fig. 6. Distribution of radiation energy recorded by the IR camera after 20000 cycles 


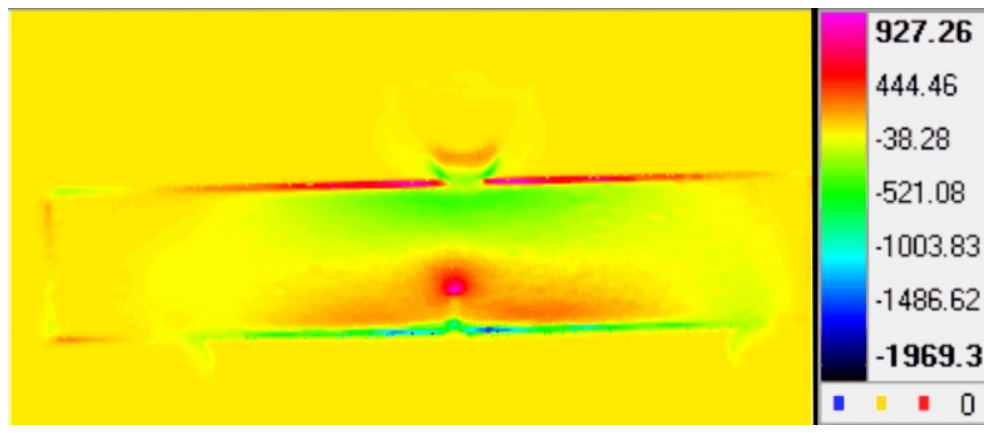

Fig. 7. Distribution of radiation energy recorded after 21500 cycles, recording made just before the expected fatigue fracture

Fatigue fracture of a sample of this material occurred after 24,149 cycles Fig. 7 . These images demonstrated the possibility of analysing the area around the crack root, based on the different radiated energy in the IR area. These differences are attributed to different energy accumulation in areas around the crack due to cyclic loading.

\section{Conclusion}

Interpretation of thermograms in this article is based on the lock-in signal processing theory. Areas are characterized by corresponding radiation, which is not evenly distributed in them. This, however, allows mapping the size of the plastic zone, respectively to study this area in more detail, since it can be assumed that the values of the detected radiation correspond to the degree of plastic area creation.

The part of the results of this work has been supported by VEGA grant No. 1/0795/16 a KEGA No. 017ŽU-4/2017.

\section{References}

1. M. Sapieta, A. Sapietová, V. Dekýš, Comparison of the thermoelastic phenomenon expressions in stainless steels during cyclic loading. Metalurgia 56, 203-206 (2017)

2. V. Vavilov, Thermal / Infrared testing. Nondestructive testing, Publishing house Spektr., 732 p. (2009)

3. X. P. V. Maldague, Theory and Practice of Infrared Technology for Nondestructive Testing. Wiley, New York (2001)

4. M. Handrik, P. Kopas, V. Baniari, M. Vaško, M. Sága, Analysis of stress and strain of fatigue specimens localised in the cross-sectional area of the gauge section testing on bi-axial fatigue machine loaded in the high-cycle fatigue region. Procedia Engineering 177, 516-519 (2017)

5. P. Pecháč, M. Sága, Controlling of local search methods' parameters in memetic algorithms using the principles of simulated annealing. Proc. Eng. 136, 70-76 (2016)

6. L. Jakubovičová, M. Sága, Computational analysis of contact stress distribution in the case of mutual stewing of roller bearing rings. Novel Trends in Production Devices and Systems, Applied Mechanics and Materials 474, 363-368 (2014)

7. J. Zapoměl, V. Dekýš, P. Ferfecki, A. Sapietová, M. Sága, M. Žmindák, Identification of material damping of a carbon composite bar and study of its effect on attenuation of its transient lateral vibrations. Int. Journal of Applied Mechanics 7 (6), (2015) 